\title{
THE EFFECTS OF A STRENGTHENING PROGRAM ON WALKING AND STAIR-CLIMBING ABILITY OF ADOLESCENTS AND YOUNG ADULTS WITH CEREBRAL PALSY: A RANDOMIZED CONTROLLED TRIAL
}

original paper

() Wroclaw University of Health and Sport Sciences

DOI: https://doi.org/10.5114/hm.2022.111177

\section{NIKOLAOS CHRYSAGIS ${ }^{1 \oplus}$, GEORGE A. KOUMANTAKIS ${ }^{1 \oplus}$, GEORGIOS THEOTOKATOS ${ }^{2 \oplus}$, EMMANOUIL SKORDILIS ${ }^{2 \oplus}$}

${ }^{1}$ Laboratory of Advanced Physiotherapy, Department of Physiotherapy, School of Health and Care Sciences, University of West Attica, Athens, Greece

${ }^{2}$ School of Physical Education and Sport Science, National and Kapodistrian University of Athens, Athens, Greece

\section{ABSTRACT}

Purpose. The study aim was to examine the effects of a 10-week school-based strengthening program on walking and stair-climbing ability of adolescents and young adults with cerebral palsy.

Methods. Overall, 35 participants, aged 12-19 years, were classified in 3 levels in accordance with the Gross Motor Function Classification System and randomly assigned to the experimental and control group. The experimental group participated in a 10-week progressive resistance exercise program during the adapted physical education class and performed functional strength exercises (sit-to-stand, lateral and front step-up), with a frequency of 3 times per week. The experimental and control groups continued their usual care at school regarding physical therapy and/or occupational therapy. Stair-climbing ability was assessed with the Timed Up and Down Stairs test and walking ability was evaluated with the $10-\mathrm{m}$ walk test and the 1-minute walk test.

Results. There was a significant between-group improvement for stair-climbing ability favouring the intervention group $\left(F=6.519, p=0.016, \eta^{2}=0.169\right)$, whereas walking ability as assessed with the 10 -m walk test and the 1 -minute walk test remained unchanged.

Conclusions. A functional strengthening program administered in adolescents and young adults with cerebral palsy may positively influence their stair-climbing ability, without any adverse effects.

Key words: locomotion, mobility, ambulation, circuit training, diplegia, tetraplegia

\section{Introduction}

Walking is a capability essential for the engagement in daily activities; it enhances social participation and overall quality of life of individuals with and without disabilities [1]. Decrements in walking ability compromise the participation in daily activities and may lead to restrictions in daily transportation, communication, access to education, recreation, and social interactions [2].

Individuals with cerebral palsy (CP) often exhibit reduced walking ability, mainly owing to weakened muscles, deficient motor control, spasticity, decreased range of motion, and low physical fitness [3, 4]. These decrements have led several researchers to examine methods to enhance walking; the most recent findings incorporate strength training [5], aquatics [6], neurodevelopmental treatment [7], treadmill training [8], etc., although the results are sometimes ambiguous.

With regard to the effect of strengthening programs on walking ability of individuals with $\mathrm{CP}$, the results are contradictory, with researchers reporting either positive or moderate findings through systematic reviews and meta-analysis studies. Park and Kim [9], for example, conducted a meta-analysis and reported positive effects of strengthening programs upon walking efficiency of CP individuals (children and adolescents). Abbaskhanian et al. [10], on the other hand, performed

Correspondence address: Nikolaos Chrysagis, Laboratory of Advanced Physiotherapy, Department of Physiotherapy, School of Health and Care Sciences, University of West Attica, Agiou Spyridonos 28, 12243 Egaleo, Athens, Greece, e-mail: nchrisagis@uniwa.gr, https://orcid.org/0000-0002-8666-5980

Received: April 5, 2021

Accepted for publication: November 22, 2021

Citation: Chrysagis N, Koumantakis GA, Theotokatos G, Skordilis E. The effects of a strengthening program on walking and stair-climbing ability of adolescents and young adults with cerebral palsy: a randomized controlled trial. Hum Mov. 2022;23(4):148-155; doi: https://doi.org/10.5114/hm.2022.111177. 
a systematic review and claimed that 'there was mediocre evidence that strength training had no effects on self-selected walking speed' (p. 2). Similarly, Anttila et al. [11] observed moderate effectiveness of strength training on self-selected walking speed and stride length among CP children. Finally, Dodd et al. [12] reported contradicting findings in a systematic review describing the impact of resistance training programs on walking speed in individuals with $\mathrm{CP}$.

Randomized clinical trials conducted in the field have reported that resistance training programs improved muscle strength without adverse effects on spasticity, while the accompanied results of the strength gain in walking ability remain non-conclusive [13]. It has been observed that a combined program of stretching and strength training had no positive effect on gait parameters such as speed, step length, gait deviation index, and 6-minute walk [13]. Liao et al. [5] found an improved walking efficiency but no positive effect on gait speed as a result of a strengthening program. On the other hand, Gillett et al. [14] stated that young individuals with CP participating in a 12-week functional anaerobic and strength training intervention improved their strength and muscle size and these gains, eventually, enhanced their agility (shuttle run test) and walking capacity scores. Kara et al. [15] revealed that a functional progressive strength and power training consisting of plyometric and balance activities that resembled everyday activities requiring rapid joint movements was an effective intervention to improve gait (1-minute walk test) in CP individuals. Van Vulpen et al. [3] found that strength and power functional training might be beneficial for the walking capacity of CP children. Similarly, Lee et al. [16] demonstrated that a 5-week strengthening program might improve gait speed and stride length in CP individuals, while Eek et al. [17] found that an individually designed strength training program improved gait parameters, such as cadence and stride length, in CP children.

In turn, ascending and descending stairs is a daily activity similar to walking with respect to the joints involved and muscular recruitment, although more demanding in the context of the range of motion, postural control, and level of strength required [18, 19]. The motor restrictions related to $\mathrm{CP}$, such as muscle weakness, deficient motor control, and spasticity, usually affect the activity of ascending and/or descending stairs and may constitute obstacles to engagement in daily activities and overall quality of life $[1,17,20]$. Our literature review revealed few studies examining the effect of resistance training programs upon the ability to climb stairs in individuals with CP. Scholtes et al.
[21] and Taylor et al. [22] introduced a 12-week resistance training program and found that, despite the improvement in muscle strength, the ability to climb stairs and walking remained unchanged among children and adolescents. Dodd et al. [23], on the other hand, used a 6-week strengthening program and reported an overall tendency for improvement concerning fast stair-climbing and walking speed in CP youngsters. Overall, the above research findings are non-conclusive, leaving the area open for future studies in the field.

Specialists employed in special schools for individuals with $\mathrm{CP}$, such as physical therapists and adapted physical educators, usually incorporate a variety of training methods, e.g. resistance exercises, functional and playful activities, or aquatics, in order to make training more pleasant and effective [3, 24]. Their goal is to minimize their students' daily restrictions and maximize engagement in daily activities and overall functionality. Keeping in mind the conflicting results regarding the effectiveness of resistance training in walking and climbing stairs among individuals with $\mathrm{CP}$, we conducted a progressive resistance program in a circuit form combined with functional elements, such us lateral step-up and playful activities. The particular intervention program has also exhibited positive results with regard to mobility, as reported in a previous paper examining other variables (Gross Motor Function Measure - dimensions D and E, sit-to-stand, lateral step-up, and Timed Up and Go test) [25]. According to the International classification of functioning, disability and health (ICF), mobility is defined as the ability of "moving by changing body position or location or by transferring from one place to another, by carrying, moving or manipulating objects, by walking, running or climbing, and by using various forms of transportation' [26].

Therefore, the aim of the present study was to examine the effects of a functional strengthening program on walking and climbing stairs in adolescents and young adults with $\mathrm{CP}$, extending the findings of our previous publication [25]. Walking and stair climbing were selected as outcome variables because they constitute elements (components) of mobility ('moving by changing body position or location or by transferring from one place to another') according to ICF [26].

\section{Material and methods}

\section{Participants}

The present study involved 35 adolescents and young adults from 4 special schools in the wider area 
of Attica, Greece. The subjects had a diagnosis of CP (diplegia or tetraplegia) and ability to follow simple commands and were classified with the Gross Motor Function Classification System (GMFCS) I-III. Additionally, they had not participated in any organized resistance training program in the previous 3 months, had no history of recent surgery or Botox injection, and did not suffer from seizures or cardiac diseases. Their demographic characteristics are published in a previous study [25].

\section{Measuring tools}

The outcome measures included the 10-m walk test (10MWT), 1-minute walk test (1MWT), and Timed Up and Down Stairs test (TUDS). According to Chrysagis et al. [27], these measures are valid, exhibit clinical utility, and can be used for a quick, low-cost assessment of functional performance.

The 10MWT evaluated the self-selected walking speed. Specifically, the participants walked in their own pace on a $14-\mathrm{m}$ walkway with or without aids. The performance was the time required to cover $10 \mathrm{~m}$, subtracting $2 \mathrm{~m}$ from the start and from the end of the walkway [27]. High test-retest reliability was reported by Wade [28].

In 1MWT, the distance covered by the participants in a single minute, in an oval track, was recorded. The subjects were not permitted to run and were instructed to use aids if needed. Previous research evidence suggested that 1MWT had high test reliability [29, 30].

TUDS assessed the ability, measured as time, of the participant to ascend and descend a staircase of 4 steps, $12 \mathrm{~cm}$ high each, with handrails on both sides. The subjects were instructed to complete the task as quickly as possible, without running, using handrails if needed. The test started with the command 'go' and completed when both feet of the participant were at the bottom of the staircase. High levels of reliability [18] and concurrent and construct validity [27] have been reported in the literature.

\section{Procedure}

The primary researcher initially informed the administrators about the purposes of the study. Accordingly, personal contact was established with the participants and their parents, and an explanation of the study details was provided. The research involved a single-blind randomized controlled trial and the assessments were conducted by 2 physiotherapists experienced in paediatric rehabilitation.
The participants were randomly assigned to the experimental and the control group, and stratified in accordance with GMFCS (GMFCS I: $n=9$, II: $n=15$, and III: $n=11$ ). The researchers used sealed envelopes specifying the experimental (I: $n=5$, II: $n=8$, III: $n=5$ ) and the control group (I: $n=4$, II: $n=7$, III: $n=6)$.

The control group continued their usual care consisting of physical therapy, occupational therapy, and adapted physical education in accordance with the curriculum. The experimental group followed their school program regarding physiotherapy and occupational therapy. None of the groups participated in any organized resistance training program during the study.

The intervention program lasted for 10 weeks, with a frequency of 3 times per week, during the adapted physical education classes, in 3 separate stations and in a circuit format. Each session lasted for approximately 30 minutes, with 5 minutes of warm-up and 5 minutes of cool-down exercises; 2-minute intervals between the activities and sets were provided. The amount of resistance was initially estimated with the 10-repetition maximum (10RM) test [21, 24, 31]. For the weekly progressions in the second and third station, $5-10 \%$ resistance was added when the participants could execute more than 12 repetitions on the third set of each activity. The subjects were all actively involved throughout the intervention, and only 2 missing sessions were recorded, due to sickness.

The first station incorporated activities such as sitto-stand, front and lateral step-up in combination with playful activities, e.g. passing and throwing a ball; the participants had an opportunity to familiarize themselves with the respective exercise patterns. The weight bearing activity (without additional load) allowed individuals to move quickly (sit-to-stand and catching a ball), depending on their capabilities. During these exercises, they had to pass or catch a ball in cooperation with a physiotherapist or to throw a ball to a basketball target, which increased their motivation. The second station consisted of the sit-to-stand activity with a backpack of weights. The load for the sit-to-stand was gradually increased as estimated by a 10RM test. Specifically, the training volume increased by $5-10 \%$ of load increments up to $60 \%$ of $10 \mathrm{RM}$ for the first 4 weeks, and up to $90 \%$ of $10 \mathrm{RM}$ for the last 6 weeks of the program. A correct performance of the exercise was a prerequisite for increasing the load. The participants, again, performed 3 sets of 10 repetitions. In the third station, they conducted sit-to-stand, as well as lateral or front step-up with low load. Specifically, in this station, the load for the lateral and front step-up was 
progressively increased from body weight to $50 \%$ of 10RM (for the last 6 weeks), with the consideration of the correct performance. The individuals carried out 3 sets of 10 repetitions for each leg; in cases of inability to execute the task, the sit-to-stand exercise was chosen.

\section{Statistical analysis}

The Statistical Package for the Social Sciences (SPSS) 20 was used for data analyses. The sample size was determined in the G*Power 3.1.9.7 software for Windows by using the minimal clinically important difference (MCID) statistic, specified from the test-retest reliability of the outcomes utilized in this study, and set as a minimum between-group difference estimate. Specifically, the TUDS measure had the smallest MCID absolute value. On the basis of this value, for TUDS MCID = 3.0 seconds between-group difference, a between-group standard deviation $(S D)$ of 3.0, a power level of 0.8 , and a significance level of $\alpha=0.05$, the minimum number of participants was calculated as $n=16$ per group.

Independent samples $t$-tests examined the pre-test (baseline) differences between the experimental and control groups regarding TUDS, 1MWT, 10MWT, and continuous demographic variables. The MannWhitney $U$ test evaluated baseline between-group differences for categorical variables. Further, intraclass correlation coefficients (ICC), standard error of measurement (SEM), and MCID were used to establish the reliability of the 3 outcome measures used in the study, via 2 within-day test-retest measurements for each outcome with a 10-minute rest between measurements. ICC values of $<0.50,0.50-0.75,0.75-0.90$, and $>0.90$ were considered to indicate poor, moderate, good, and excellent reliability, respectively. The associated SEM $=S D \sqrt{ } 1-\mathrm{ICC}$ and $\mathrm{MCID}_{95 \% \mathrm{CI}}=1.96 \sqrt{2} \mathrm{SEM}$ were calculated for each ICC [32]. MCID represents the minimum amount of change that can be considered a real one, over and above the error of measurement [32].

Finally, separate ANCOVAs were used to examine post-test differences for TUDS, 1MWT, and speed (10MWT) between the experimental and control groups. ANCOVA analysis is preferentially applied in interventional experimental designs to remove extraneous influences, such as pre-test differences [33, 34]. The statistical significance of the results was accepted at $p<0.05$.

\section{Ethical approval}

The research related to human use has complied with all the relevant national regulations and institutional policies, has followed the tenets of the Declaration of Helsinki, and has been approved by the ethics committee of the National and Kapodistrian University of Athens and the Greek Ministry of Education.

\section{Informed consent}

Informed consent has been obtained from all individuals included in this study and/or their legal guardians.

\section{Results}

Demographic and baseline data comparisons are shown in Table 1, with none of the comparisons between the experimental group and the control group presenting significant differences. Accordingly, testretest reliability data are depicted in Table 2, with all 3 variables demonstrating good or excellent reliability. Pre- and post-test data of all outcomes are provided in Table 3 and those for TUDS are graphically presented in Figure 1.

The ANCOVAs revealed significant post-test differences, with pre-test as a covariate, with respect to TUDS $\left(F=6.519, p=0.016, \eta^{2}=0.169\right)$. The adjusted post-test mean score of the experimental group was significantly lower than in the control group. The effect size for this measure was large $\left(\eta^{2}>0.14\right)$ [32]. In turn, no significant differences were found for 1MWT $(F=$ $\left.1.520, p=0.226, \eta^{2}=0.046\right)$ or speed $(F=0.602, p=$ $\left.0.443, \eta^{2}=0.018\right)$. The ANCOVA results are presented in Table 4.

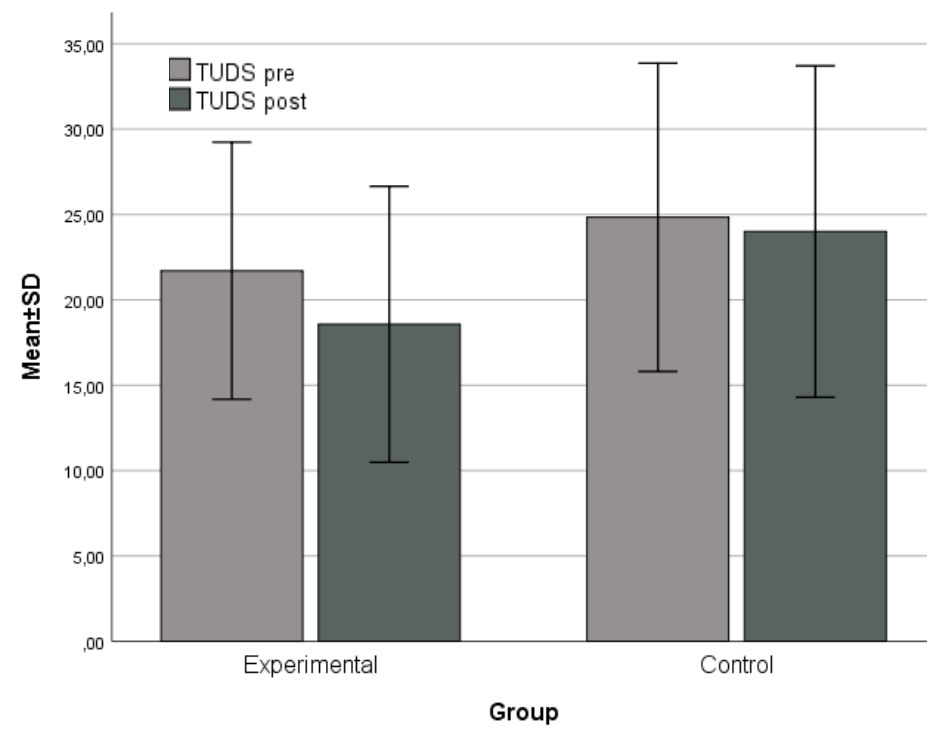

Figure 1. Pre- and post-test data for the Timed Up and Down Stairs test (TUDS) 


\section{HUMAN MOVEMENT}

N. Chrysagis et al., Strengthening program for cerebral palsy

Table 1. Baseline comparisons with the independent samples $t$-test for continuous variables (mean \pm standard deviations) or the Mann-Whitney $U$ test for categorical variables regarding the anthropometric, clinical condition classification, and functional testing values

\begin{tabular}{lccc}
\hline Variables & Experimental group & Control group & $p$ \\
\hline Age (years) & $15.27 \pm 2.10$ & $14.70 \pm 1.89$ & 0.406 \\
Muscle mass (kg) & $53.30 \pm 7.27$ & $51.47 \pm 5.46$ & 0.407 \\
Height (cm) & $159.11 \pm 7.37$ & $156.23 \pm 6.00$ & 0.216 \\
GMFCS I/II/III ( $n)$ & $5 / 8 / 5$ & $4 / 7 / 6$ & 0.656 \\
Limbs affected: diplegia/tetraplegia $(n)$ & $15 / 3$ & $13 / 4$ & 0.384 \\
10-m walk test (m/min) & $50.70 \pm 10.46$ & $46.23 \pm 13.39$ & 0.279 \\
1-minute walk test (m) & $74.12 \pm 24.18$ & $64.45 \pm 24.11$ & 0.245 \\
Timed Up and Down Stairs test (s) & $21.70 \pm 7.52$ & $24.83 \pm 9.03$ & 0.272 \\
\hline
\end{tabular}

GMFCS - Gross Motor Function Classification System

Table 2. Mean and standard deviation values of the same-day test-retest reliability procedure and relevant relative (ICC) and absolute (SEM/MCID) reliability outcomes $(n=35)$

\begin{tabular}{lcccrr}
\hline Variables & $1^{\text {st }}$ measurement & $2^{\text {nd }}$ measurement & ICC (95\% CI) & SEM & MCID $95 \%$ CI \\
\hline TUDS (s) & $23.2 \pm 8.3$ & $23.7 \pm 7.3$ & $0.845(0.81-0.87)$ & 1.1 & 3.0 \\
1MWT (m) & $69.4 \pm 24.3$ & $70.1 \pm 23.9$ & $0.920(0.89-0.95)$ & 2.2 & 6.1 \\
10MWT (m/min) & $48.5 \pm 12.0$ & $49.0 \pm 11.8$ & $0.979(0.96-0.99)$ & 3.9 & 10.7 \\
\hline
\end{tabular}

ICC - intraclass correlation coefficient, SEM - standard error of measurement, MCID - minimal clinically important difference, TUDS - Timed Up and Down Stairs test, 1MWT - 1-minute walk test, 10MWT - 10-m walk test

Table 3. Pre- and post-test data for the outcome variables

\begin{tabular}{lcc}
\hline Variables & Pre-test & Post-test \\
\hline Timed Up and Down Stairs test (s) & & \\
$\quad$ Experimental group & $21.70 \pm 07.52$ & $18.57 \pm 08.08$ \\
$\quad$ Control group & $24.83 \pm 09.03$ & $24.00 \pm 09.70$ \\
\hline 1-minute walk test (m) & & \\
Experimental group & $74.12 \pm 24.18$ & $77.40 \pm 23.91$ \\
Control group & $64.45 \pm 24.11$ & $65.27 \pm 24.61$ \\
\hline 10-m walk test (m/min) & & \\
Experimental group & $50.70 \pm 10.46$ & $53.68 \pm 12.48$ \\
Control group & $46.23 \pm 13.39$ & $47.61 \pm 13.92$ \\
\hline
\end{tabular}

Table 4. ANCOVA findings for the outcome variables

\begin{tabular}{lccc}
\hline Variables & Adjusted post-test & $F$ & $p$ \\
\hline TUDS & & & \\
$\quad$ Experimental group & 20.14 & 6.519 & 0.016 \\
$\quad$ Control group & 22.34 & & 0.169 \\
1MWT & & & 0.226 \\
$\quad$ Experimental group & 72.79 & 1.520 & \\
$\quad$ Control group & 70.61 & & 0.046 \\
10MWT & 51.86 & 0.602 & 0.443 \\
$\quad$ Experimental group & 49.54 & & 0.018 \\
$\quad$ Control group & & & \\
\hline
\end{tabular}

TUDS - Timed Up and Down Stairs test, 1MWT - 1-minute walk test, 10MWT - 10-m walk test 


\section{Discussion}

The present findings suggest that a 10 -week functional strengthening program implemented during adapted physical education classes improved the ability to climb stairs (TUDS) in CP adolescents and young adults. The program, however, did not appear to have an effect upon the walking ability (1MWT, 10MWT) of the individuals involved. Further, Chrysagis et al. [25] previously reported that the particular program applied in this sample of patients had an effect upon the lower limb strength, with no adverse effects regarding passive range of motion or spasticity. Specifically, a significant effect was previously observed for the isometric strength of knee extensors and hip abductors but not for knee flexors. Accordingly, no changes were detected with respect to the passive range of motion (of knee extensors and hip abductors) or spasticity (of hip adductors, knee flexors, and knee extensors) [25].

The present observations are in agreement with those by Dodd et al. [23], who reported an improvement (although non-significant) in stair climbing after the completion of a 6-week home-based functional strengthening program in CP youngsters. Scholtes et al. [21] and Taylor et al. [22], however, found that progressive strength training had no effect on climbing stairs in adolescents and children with $\mathrm{CP}$, despite the positive effect upon strength. The differences with the present study may be due to the samples employed, which differed with regard to age, type of disability (hemiplegia, diplegia), intervention program, and statistical analysis used. Taylor et al. [22], for example, applied exercise machines to conduct the progressive strengthening program, while in the present study, functional taskspecific exercises were used. Furthermore, Scholtes et al. [21] examined the effect of progressive strengthening in a sample of children with hemiplegia and diplegia.

With respect to the walking ability, assessed with the 10MWT and 1MWT measures, the present findings are in line with Scholtes et al. [21], Taylor et al. [22], and Unger et al. [35]. Scholtes et al. [21] conducted 10 -week functional progressive resistance training in a circuit format among children with $\mathrm{CP}$ in a school environment. They reported that the experimental group had not improved significantly concerning the walking speed, step length, or cadence compared with the control group. Similarly, Taylor et al. [22] found that a 12-week progressive resistance program had exerted no effect on the walking ability measured with 1MWT and 10MWT among CP youngsters. Unger et al. [35] observed no significant change of walking speed, ca- dence, or stride length as a result of an 8-week strengthening program. On the other hand, a trend for improvement in walking ability was reported [23], but this trend was not supported by post-test differences between the experimental and control group after a 6 -week strengthening program. A tendency for improvement in gait parameters, although without significant differences between groups, was also demonstrated after a postoperative strength training program [36]. Similarly, in the present study, the non-significant improvement regarding walking ability may be explained by the dissimilar tasks employed in relation to walking [13], specific type of the exercises performed [22], or a ceiling effect of strength, which was essential for walking [21]. The exercises implemented in this study (sit-to-stand and lateral step-up), although contributing to strength improvement of knee extensors and hip abductors, did not seem to have a carry-over effect on walking ability, since walking exercises were not included in the intervention protocol. Further, given the specificity of training principles, a transferability effect could be present if task-specific exercises such as gait training on a treadmill were included.

On the other hand, recently published randomized clinical trials have reported that strengthening programs combined with power $[3,15]$ and anaerobic training [14] may have a positive effect upon the walking ability of individuals with CP. In line with those findings, Lee et al. [16] suggested that strengthening, including functional exercises with free weights and bicycle isokinetic training, improved gait speed and stride length, as well as decreased the double support phase in CP children.

Ascending and descending stairs are activities that differ from walking with regard to the biomechanics and the effort necessary to complete the task. Specifically, a larger amount of strength, a certain range of motion, and postural control are needed to move the body up and down stairs [19]. The ability to climb stairs was significantly enhanced and this change may be due to the improved strength scores for the knee extensors and hip abductors, previously reported for this population elsewhere [25]. This argument is in line with the principles of training specificity, claiming that there is a direct relationship between movement patterns and the testing used [37], and the result of specific strengthening exercises (e.g. lateral and front stepup) may be transferred to similar activities, such as climbing stairs [38].

The present study is subject to certain limitations which do not allow for generalizations without caution. These limitations have been reported by Chrysagis et 
al. [25] and mainly incorporate the classification levels, convenience sampling, and the participation in the usual physical therapy and/or occupational therapy sessions and additional adapted physical activities during the program. The subjects classified in GMFCS II or III, for example, often held the handrails during testing, while those classified in GMFCS I did not; those classified as GMFCS level III performed 10MWT and the 1MWT using a walker $(n=7)$ or sticks $(n=4)$. Motivation, variability of neuromuscular responses to resistive exercise owing to spasticity or motor control deficits, and missing sessions of the participants could constitute further limitations.

The present study shows that the functional strengthening program had positive effects on the stairclimbing ability of adolescents and young adults with CP. The involvement in functional and playful resistance exercises enhanced the ascending and descending stairs ability, and these gains became evident for the participants. Overall, the present study supports the idea that step-up and -down exercises with extra resistance or with body weight and functional and playful activities may be included in the adapted physical education and physical therapy programs.

Future research may evaluate the effect of long-term strengthening programs on other parameters, such us psychosocial condition, activities of daily living, and quality of life, of individuals with CP. Additionally, the effect of the above programs should be examined separately for different levels of GMFCS or individually established pre-existing muscle (such us dorsiflexors) weakening. Finally, studies with high methodological quality taking into account the heterogeneity and complexity of individuals with $\mathrm{CP}$ are strongly recommended.

\section{Conclusions}

The present study revealed that a functional strengthening program improved stair-climbing ability of adolescents and young adults with CP. Walking ability, however, remained unchanged after the intervention. Task-specific exercises, e.g. on a treadmill, may be used instead to enhance walking-related variables such us speed or distance covered in a specific time.

\section{Disclosure statement}

No author has any financial interest or received any financial benefit from this research.

\section{Conflict of interest}

The authors state no conflict of interest.

\section{References}

1. Gjesdal BE, Jahnsen R, Morgan P, Opheim A, Mæland S. Walking through life with cerebral palsy: reflections on daily walking by adults with cerebral palsy. IntJQualStudHealthWell-being. 2020;15(1):1746577; doi: 10.1080/17482631.2020.1746577.

2. Østensjø S, Brogren Carlberg E, Vøllestad NK. Motor impairments in young children with cerebral palsy: relationship to gross motor function and everyday activities. Dev Med Child Neurol. 2004;46(9):580-589; doi: 10.1017/s0012162204000994.

3. Van Vulpen LF, de Groot S, Rameckers E, Becher JG, Dallmeijer AJ. Improved walking capacity and muscle strength after functional power-training in young children with cerebral palsy. Neurorehabil Neural Repair. 2017;31(9):827-841; doi: 10.1177/1545968317723750.

4. Graham HK, Rosenbaum P, Paneth N, Dan B, Lin J-P, Damiano DL, et al. Erratum: cerebral palsy. Nat Rev Dis Primers. 2016;2:16005; doi: 10.1038/nrdp.2016.5.

5. Liao H-F, Liu Y-C, Liu W-Y, Lin Y-T. Effectiveness of loaded sit-to-stand resistance exercise for children with mild spastic diplegia: a randomized clinical trial. Arch Phys Med Rehabil. 2007;88(1):25-31; doi: 10.1016/j. apmr.2006.10.006.

6. Chrysagis N, Douka A, Nikopoulos M, Apostolopoulou F, Koutsouki D. Effects of an aquatic program on gross motor function of children with spastic cerebral palsy. Biol Exerc. 2009;5(2):13-25; doi: 10.4127/jbe. 2009.0027.

7. Kavlak E, Ünal A, Tekin F, Altuğ F. Effectiveness of Bobath therapy on balance in cerebral palsy. Cukurova Med J.2018;43(4):975-981; doi: 10.17826/cumj.375565.

8. Chrysagis N, Skordilis EK, Stavrou N, Grammatopoulou E, Koutsouki D. The effect of treadmill training on gross motor function and walking speed in ambulatory adolescents with cerebral palsy: a randomized controlled trial. Am J Phys Med Rehabil. 2012;91(9): 747-760; doi: 10.1097/PHM.0b013e3182643eba.

9. Park E-Y, Kim W-H. Meta-analysis of the effect of strengthening interventions in individuals with cerebral palsy. Res Dev Disabil. 2014;35(2):239-249; doi: 10.1016/j.ridd.2013.10.021.

10. Abbaskhanian A, Rashedi V, Delpak A, Vameghi R, Gharib M. Rehabilitation interventions for children with cerebral palsy: a systematic review. J Pediatr Rev. 2015;3(1):e361; doi: 10.5812/jpr.361.

11. Anttila H, Autti-Rämö I, Suoranta J, Mäkelä M, Malmivaara A. Effectiveness of physical therapy interventions for children with cerebral palsy: a systematic review. BMC Pediatr. 2008;8(1):14; doi: 10.1186/14712431-8-14.

12. Dodd KJ, Taylor NF, Damiano DL. A systematic review of the effectiveness of strength-training programs for people with cerebral palsy. Arch Phys Med Rehabil. 2002; 83(8):1157-1164; doi: 10.1053/apmr.2002.34286.

13. Aarsland Fosdahl M, Jahnsen R, Kvalheim K, Holm I. Effect of a combined stretching and strength training 
program on gait function in children with cerebral palsy, GMFCS level I \& II: a randomized controlled trial. Medicina. 2019;55(6):250; doi: 10.3390/medicina55 060250.

14. Gillett JG, Lichtwark GA, Boyd RN, Barber LA. Functional anaerobic and strength training in young adults with cerebral palsy. Med Sci Sports Exerc. 2018;50(8): 1549-1557; doi: 10.1249/MSS.0000000000001614.

15. Kara OK, Livanelioglu A, Yardımcı BN, Soylu AR. The effects of functional progressive strength and power training in children with unilateral cerebral palsy. Pediatr Phys Ther. 2019;31(3):286-295; doi: 10.1097/ PEP.0000000000000628.

16. Lee JH, Sung IY, Yoo JY. Therapeutic effects of strengthening exercise on gait function of cerebral palsy. Disabil Rehabil. 2008;30(19):1439-1444; doi: 10.1080/09638 280701618943.

17. Eek MN, Tranberg R, Zügner R, Alkema K, Beckung E. Muscle strength training to improve gait function in children with cerebral palsy. Dev Med Child Neurol. 2008;50(10):759-764; doi:10.1111/j.1469-8749.2008. 03045.x.

18. Zaino CA, Marchese VG, Westcott SL. Timed up and down stairs test: preliminary reliability and validity of a new measure of functional mobility. Pediatr Phys Ther. 2004;16(2):90-98; doi: 10.1097/01.PEP.000012 7564.08922.6A.

19. Bar-Haim S, Belokopytov M, Harries N, Frank A. A stairclimbing test for ambulatory assessment of children with cerebral palsy. Gait Posture. 2004;20(2):183-188; doi: 10.1016/j.gaitpost.2003.09.002.

20. Wichers M, Hilberink S, Roebroeck ME, van Nieuwenhuizen O, Stam HJ. Motor impairments and activity limitations in children with spastic cerebral palsy: a Dutch population-based study. J Rehabil Med. 2009; 41(5):367-374; doi: 10.2340/16501977-0339.

21. Scholtes VA, Becher JG, Janssen-Potten YJ, Dekkers H, Smallenbroek L, Dallmeijer AJ. Effectiveness of functional progressive resistance exercise training on walking ability in children with cerebral palsy: a randomized controlled trial. Res Dev Disabil. 2012;33(1):181-188; doi: 10.1016/j.ridd.2011.08.026.

22. Taylor NF, Dodd KJ, Baker RJ, Willoughby K, Thomason P, Graham HK. Progressive resistance training and mobility-related function in young people with cerebral palsy: a randomized controlled trial. Dev Med Child Neurol. 2013;55(9):806-812; doi: 10.1111/dmcn. 12190.

23. Dodd KJ, Taylor NF, Graham HK. A randomized clinical trial of strength training in young people with cerebral palsy. Dev Med Child Neurol. 2003;45(10):652657; doi: 10.1017/s0012162203001221.

24. Scholtes VA, Becher JG, Comuth A, Dekkers H, Van Dijk L, Dallmeijer AJ. Effectiveness of functional progressive resistance exercise strength training on muscle strength and mobility in children with cerebral palsy: a randomized controlled trial. Dev Med Child Neurol. 2010;52(6):e107-e113;doi:10.1111/j.1469-8749.2009. 03604.x.

25. Chrysagis N, Skordilis E, Grammatopoulou E, Douka A. The effect of a functional progressive strength training program on mobility of ambulatory adolescents and young adults with cerebral palsy. Sports Med Rehabil J. 2019;4(1):1046.

26. World Health Organization. International classification of functioning, disability and health (ICF). Available from: https://www.who.int/classifications/internationalclassification-of-functioning-disability-and-health.

27. Chrysagis N, Skordilis EK, Koutsouki D. Validity and clinical utility of functional assessments in children with cerebral palsy. Arch Phys Med Rehabil. 2014;95(2): 369-374; doi: 10.1016/j.apmr.2013.10.025.

28. Wade DT. Measurement in neurological rehabilitation. New York: Oxford University Press; 1992.

29. McDowell BC, Humphreys L, Kerr C, Stevenson M. Testretest reliability of a 1-min walk test in children with bilateral spastic cerebral palsy (BSCP). Gait Posture. 2009;29(2):267-269; doi:10.1016/j.gaitpost.2008.09.010.

30. McDowell BC, Kerr C, Parkes J, Cosgrove A. Validity of a 1 minute walk test for children with cerebral palsy. Dev Med Child Neurol. 2005;47(11):744-748; doi: 10.1017/S0012162205001568.

31. Baechle TR, Earle RW. Essentials of strength training and conditioning, $3^{\text {rd }}$ ed. Champaign: Human Kinetics; 2008.

32. Portney LG, Watkins MP. Foundations of clinical research: applications to practice, $3^{\text {rd }}$ ed. Harlow: Pearson Education Ltd., 2014.

33. Tabachnick BG, Fidell LS. Using multivariate statistics, $4^{\text {th }}$ ed. Boston: Allyn and Bacon; 2001.

34. Thomas JR, Nelson JK, Silverman SJ. Research methods in physical activity, $5^{\text {th }}$ ed. Champaign: Human Kinetics; 2005.

35. Unger M, Faure M, Frieg A. Strength training in adolescent learners with cerebral palsy: a randomized controlled trial. Clin Rehabil. 2006;20(6):469-477; doi: 10.1191/0269215506cr961oa.

36. Patikas D, Wolf SI, Mund K, Armbrust P, Schuster W, Döderlein L. Effects of a postoperative strength-training program on the walking ability of children with cerebral palsy: a randomized controlled trial. Arch Phys Med Rehabil. 2006;87(5):619-626; doi: 10.1016/j. apmr.2006.01.023.

37. Gamble P. Implications and applications of training specificity for coaches and athletes. Strength Cond J. 2006;28(3):54-58; doi: 10.1519/1533-4295(2006)28 [54:IAAOTS]2.0.CO;2.

38. Carr JH, Shepherd RB. Neurological rehabilitation: optimizing motor performance. Oxford: ButterworthHeinemann; 1998. 